\title{
Use of Carbohydrate Antigen 19-9 in the management of bladder cancer
}

\author{
Mr. Muhammad Faisal Khan, Tsampoukas Georgios
}

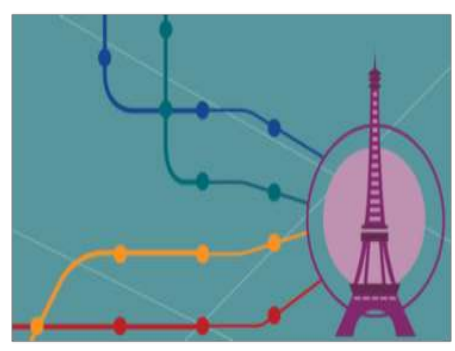

\section{INTRODUCTION:}

Bladder cancer (BC) is the 11th most common cancer in the general population of Europe, with the worldwide age-standardized mortality rate (per 100.000 persons/year) is estimated at 3.2 for men and 0.9 for women, respectively. Over the years, a distant effort by researchers has been made to provide new prognostic biomarkers in the assessment of accurate response to treatment and prompt detection of recurrences. Among several markers, the carbohydrate-rich glycoprotein, Carbohydrate Antigen (CA) 19-9 has shown some usefulness as it is linked with the tumour aggressiveness and the prognosis of the disease.

\section{METHODS:}

\section{Evidence acquisition:}

\section{Inclusion criteria}

- Studies reporting on patients with bladder cancer and carbohydrate antigen 19-9 measurement

- Prospective studies written in the English language from inception to August 2019.

\section{Other criteria*}

- Case series

- Non-urothelial malignancies such as colorectal malignancy

- Benign urological diseases

* These criteria were mentioned in the article to evaluate the established role of Carbohydrate antigen 19-9.

\section{Search strategy}

The systematic review was performed according to the Cochrane reviews guidelines and the Preferred Reporting Items for Systematic Reviews and Meta-Analyses (PRISMA) guidelines. We searched PubMed, Embase and Cochrane Library from inception to August 2019, and all English language articles were included in the original search. The search terms included; Bladder cancer; Urothelial cancer; Carbohydrate antigen 19-9 and CA 19-9; Boolean operators (AND, OR) were used with the above search terms to refine the search procedure.

\section{CONCLUSIONS:}

Urinary levels of carbohydrate antigen 19- 9 seem to carry some significance for the detection of low grade tumours In comparison to cytology and the modality looks promising. However, It should be kept in mind that Urinary levels of the biomarker may be elevated in Urinary tract obstruction, which could limit its role in some patients . Furthermore, what really matters In Patients with nonmuscle Invasive tumours is grade, as this is the factor that dictates prognosis. If the modality should be combined with cytology in order to substitute or assist or avoid unnecessary cystoscopies for the diagnosis or follow-up of bladder cancer, it could be a matter of future, prospective studies. However, no evidence exists yet, that the use of the marker can be recommended. Secondly, another common problem in bladder cancer is clinical under staging or over staging, which addressed to low accuracy of staging modalities (bimanual examination, Computer Tomography or Magnetic Resonance Imaging). In previous studies, Margel et al have demonstrated that increased serum levels of CA 19-9 in patients who underwent cystectomy for localized disease (T2NOMO) were high risk of extravesical and nodal positive disease; the patients in that setting had inevitably reduced survival. Although the patients in those cohorts did not receive neoadjuvant chemotherapy, data implicates that the biomarkers like CA 19-9 might bridge the gap between clinical and pathological staging, avoiding unnecessary interventions and alternate management towards the most feasible outcome. Similarly, in an aforementioned study, patients who received neoadjuvant chemotherapy and had their biomarkers normalized, enjoyed significantly longer overall survival and lower recurrence, although the pathological stage did not differ between responders and nonresponders. These results should be considered significant, as far as the biomarker might provide a way to unmask patients at highest risk and alternate the management of the condition for the benefit of these patients. Under these circumstances, CA 19-9 could play a role in bladder cancer similar to that in pancreatic carcinoma; having a limited role in diagnosis and should the diagnosis of the urothelial carcinoma has been set, serum levels can be measure of tumour aggressiveness and help in the monitoring of the disease. However, pitfalls may occur. As far as tumours poorly differentiated may not express CA 19-9, indicating an unpredictably high malignant potential.

\begin{tabular}{|l|l|}
\hline Pectasides et al. & $\begin{array}{l}\text { CA 19-9 is a potential tool for monitoring the } \\
\text { efficacy of treatment of TCC bladder. }\end{array}$ \\
\hline Vestergaard et al. & $\begin{array}{l}\text { Further investigations are needed to find } \\
\text { usefulness of CA19-9. }\end{array}$ \\
\hline Cook et al. & CA 19-9 can predict response to chemotherapy \\
\hline Sashide et al. & CA 19-9 is useful for prognosis \\
\hline Margel et al. & CA 19-9 can predict disease specific mortality \\
\hline Nagao et al. & CA 19-9 can be effective diagnostic tool \\
\hline Margel et al. & CA 19-9 85\% accurate in predicting metastasis. \\
\hline Hegele et al & $\begin{array}{l}\text { CA 19-9 not accurate for diagnosis but for } \\
\text { tumour invasion \& grading. }\end{array}$ \\
\hline
\end{tabular}

\begin{tabular}{|l|l|}
\hline Taga et al. & $\begin{array}{l}\text { CA19-9 plays important role in predicting } \\
\text { prognosis. }\end{array}$ \\
\hline Pal et al. & CA19-9 may be useful for diagnosis \\
\hline Jha et al. & CA19-9 high levels predicts poor prognosis \\
\hline Roy et all. & CA19-9 better prognostic marker. \\
\hline Ahmadi et al. & $\begin{array}{l}\text { CA19-9 high levels pre cystectomy predicts a } \\
\text { poor prognosis. }\end{array}$ \\
\hline Wang et al. & $\begin{array}{l}\text { CA19-9 not good for diagnosis but strong marker } \\
\text { for prognosis. }\end{array}$ \\
\hline Bazargani et al. & $\begin{array}{l}\text { CA19-9 persistently high levels after chemo } \\
\text { predict poor outcome after cystectomy }\end{array}$ \\
\hline Yaegashi et al. & $\begin{array}{l}\text { CA19-9 may predict good prognosis in Patients } \\
\text { with advanced UC }\end{array}$ \\
\hline
\end{tabular}

